\title{
INITIAL EUPHORIA TO SUSTAINED CHANGE - MAINSTREAMING LEAN CULTURE
}

\author{
Venkata S. K. Delhi ${ }^{1}$, Raghavan $\mathrm{N}^{2}$, Ashwin Mahalingam ${ }^{3}$, Koshy Varghese ${ }^{4}$
}

\begin{abstract}
In line with the trend of spread of Lean Construction concepts in the recent past, Lean construction philosophy was introduced in a large industrial project of a leading organisation about two years back. The drive by the top management, augmented by efforts of the Lean champions in the project team, helped the project team to transition from the conventional project delivery method to the Lean Construction method. The present study looks at the developments in Lean implementation in the Project over time, based on observations on such dynamics and understanding the strategies which might help to mainstream Lean philosophy introduction in other similar organisations and construction projects.

A detailed case study based approach was adopted to this end. The mentoring coach closely observed the behavioural developments of the Project team over time. Data for analysis included primary observations by the mentoring coach, minutes of the various meetings of the project team under study and one- on- one interactions with various team members. The analysis gave some significant insights on how to continue the initial momentum gained towards sustained Lean implementation on construction projects and in organisations.
\end{abstract}

Keywords: Sustained Lean Practice, Lean Culture, Transformation, Behaviour change, Human resources.

\section{INTRODUCTION}

Lean construction has gained some momentum in India over the last decade. . The formation of bodies like ILCE (Institute for Lean Construction Excellence) and research initiatives from leading educational institutions such as Indian Institute of Technology Madras (IITM) helped in securing institutional support for the organisations willing to transform and adopt Lean philosophy. To this end, many such construction organisations are testing Lean construction concepts on pilot basis on some of their construction projects. The initial gains witnessed from such projects include reduction in wastage, time and cost benefits, improvement in the morale of the teams involved among many other benefits (Kaiser, 2012; Raghavan, 2015). Such witnessed benefits encouraged these organisations to attempt to institutionalise Lean practices in their organisational processes. One such commendable attempt by a leading corporate is reported in this paper.

1 Assistant Professor, Civil Engineering Dept., Indian Institute of Technology Bombay, Mumbai, India, venkatad@iitb.ac.in

2 Professor of Practice, Dept. of Civil Engineering, Indian Institute of Technology Madras, Chennai, India nraghavan@iitm.ac.in

3 Associate Professor, Dept. of Civil Engineering, Indian Institute of Technology Madras, Chennai, India, mash@iitm.ac.in

4 Professor, Dept. of Civil Engineering, Indian Institute of Technology Madras, Chennai, India koshy@iitm.ac.in 
In this context, this large conglomerate with a distinguished long history had initiated the introduction of Lean philosophy in some of its construction activities., After a couple of successful trial implementations conducted in a training mode, the organisation decided to introduce Lean construction in a large industrial project on a pilot basis. The initial part of how Lean construction philosophy was introduced and the learning from the initial exercise were reported in an earlier paper (Raghavan et. al. 2016). An external mentoring coach team which was hired to give guidance on Lean implementation decided to use this unique opportunity to study the various barriers and facilitators to mainstream Lean on a long term basis in a Project, and then further on in the overall organisation.

\section{OBJECTIVES OF THE STUDY}

Further to the earlier study which looked at the initial learning curve, the present study is aimed at understanding the organisational dynamics and other factors which help in sustaining the initial momentum towards comprehensive implementation of Lean construction in a project and in the organisation.

\section{RESEARCH METHOD}

To address these objectives, a detailed case- based research approach was adopted in this study. After the initial take-off the mentoring coach observed the sustained practice of Lean concepts in the project as well as in the organisation. The mentoring coach also observed the development of Lean culture in the various construction teams over time. Data for analysis included primary observations by the research team, minutes of meetings of the project team under study, a survey questionnaire addressed to the team members and one- on- one interactions with various team members. The various observations made from time to time were analysed by the mentoring coach and corrective courses were communicated to the project teams for steady progress on the Lean road. Decisions were made on the basis of data collected from different methods/ sources and not from any subjective view points.

\section{CASE STUDY - INDUSTRIAL PROJECT}

The new industrial project included Engineering, Procurement and Construction phases. The parent organisation was basically a diversified, highly reputed manufacturing company and it also had a construction division. The construction division had the responsibility to construct and deliver this crucial project to its parent organisation. The top management of the construction division sensed this as a good opportunity to introduce Lean construction on the project. The division had tried out Lean construction earlier on pilot projects and had tasted some success. Hence the division wanted to try this philosophy on a project with larger scope. The top management hoped that the new construction philosophy would help them save time and make construction more efficient across all their construction projects by and by.

As the project was being conceptualized and designed, Lean construction philosophy was introduced progressively in all the phases of the project. Various techniques such as 5S, Big Room, Last Planner SystemTM, PPC (Plan Percent Completed) monitoring, etc. were introduced progressively in the project. The team started using these tools and also started progressively adopting Lean as a work philosophy as well. 
As time progressed, the project emphasis shifted from design to construction stage. In the various stages the project encountered a number of challenges. Initially the project faced some challenges due to permits, approvals and land acquisitions which impacted the sequence and timing of development significantly. Later there were other challenges arising from various scope changes from end users, design changes, variations in soil strata, inclement weather conditions, etc. As the challenges began to unfold, the mentoring coach observed how the project team responded to these challenges and tried to understand whether the adoption of Lean philosophy helped or deterred the team from achieving their objectives.

\section{OBSERVATIONS ON SUSTAINING LEAN PHILOSOPHY}

The main observations relating to sustaining the Lean philosophy on the project and mainstreaming it in the organisation are covered in three different categories in this section - (i) Organizational structure, (ii) Procedural aspects and (iii). Organizational behaviour and culture.

\subsection{Organizational structure}

The first set of observations relates to the unique aspects pertaining to the organisational structure and how they contributed/challenged in sustaining the Lean philosophy.

\subsubsection{Sustaining the changes in organizational structure}

The traditional time-honoured (matrix) structure of this organization had multiple reporting streams for many people. For example, the manager responsible for installing MEP components of the project reported to the project manager as well as the division head of the MEP division of the company. Similarly, many team members were working on various other projects and had multiple heads to report to. The introduction of Lean exposed these complexities in the organizational structure. The regular practice of the Big Room concept and the Collaborative Planning System adopted, as well as better understanding of the inter-dependencies of the various teams, helped overcome such difficulties related to the structure. Basically people started to reach out to the concerned people directly and collaborate along lateral lines rather than along vertical lines of communications. Teams started accepting the complexities and learned to function effectively despite the challenges. Realisation of this initial benefit helped the team members appreciate the benefits of Lean and sustain Lean in the project.

Another challenge overcome by Lean practices related to compartmentalisation and lack of sharing. The prevailing planning structure before Lean was highly centralized. The project planning was done by a central team and the scopes and deadlines were determined by this team and "pushed" on to the other teams. The introduction of Lean transformed this relationship to that of a "pull" environment where the micro planning and scheduling for activities were now done by the respective team members and the central planning team aggregated these plans and checked for conformance to the broader milestones. The initial transition was quick. However, as the project progressed, there was always a tendency to swing back to the age-old planning mechanism. The benefits of initial Lean implementation weighed against the resistance to switch from a known process and this was a key dynamic which assisted long term adoption of Lean philosophy on the project. 


\subsubsection{Involvement of the top management}

During the initial phases the top management of the construction division was closely following the team's transition to Lean construction and provided considerable motivation. The management was regularly involved in the Big Room meetings during the initial phase. As the management saw that Lean was being successfully adopted on the project, its focus shifted to other pressing matters. As the top management's frequency of participation in the Lean meetings decreased, the initial enthusiasm towards Lean started waning. The behaviour of the various teams changed to some extent once they realised that they were no longer under the direct watch of the top management. This could relate somewhat to the concept of the Hawthorne effect (Landsberger, 1958), wherein the behaviour of people changed once they realised that they were being observed. This highlighted the need for the involvement of the top management to continue for a considerable period to sustain the transition to Lean construction.

\subsubsection{The changes in Lean championship team}

The initial transition to Lean methods was championed by a Lean championship team which was familiar with Lean methods from association with earlier Lean trials and which had also fully subscribed to the Lean philosophy. However, as the project progressed, some of the members of this championship team were shifted to other assignments. The dilution in the driving force provided by the champions started cooling off the interest in Lean and commitment to Lean started wavering to some extent. The learning was that the core championship team should stay with the Project for sustained momentum.

\subsection{Procedural Aspects}

The introduction of Lean philosophy involved some significant changes to the traditional processes adopted in the project. This section discusses some aspects of the processes which the Lean implementation challenged.

\subsubsection{The transparency required in organisational processes}

An interesting change in the organisational processes came about due to increased transparency in the interaction processes associated with Lean culture. Lean introduced the need to accept the project performance as paramount as against the performances of individual divisions/ teams. The use of the Big Room concept encouraged the various teams to start having conversations directly with the other teams to seek clarifications instead of going through time and energy-consuming traditional hierarchical routes. Such dynamics observed on this project point to the fact that for successful Lean implementation, the organisation should enable processes which are adequately transparent and inclusive and encourage the various players to share information among all team members including subcontractors.

\subsubsection{Proactive determination of challenges}

The use of Big Room meetings and Look Ahead schedules encouraged the team members to look at the potential constraints more proactively and seek timely and coordinated action plans. This meant introduction of new processes in the organisation which would facilitate such proactive working. Also, a new software tool was deployed to keep track of the promises and new protocols were drafted with regard to the communication and escalation mechanisms. As the project progressed, the teams were initially viewing these 
as constraining them from their usual way of doing business. However, as the project started facing various challenges (described in Section 4 above) these new processes helped the teams to navigate the challenges better. The formation of a multi-disciplinary, multi-divisional Steering Committee helped considerably in sorting out interdepartmental coordination issues, etc. The team was able to anticipate some of these challenges and the new Lean processes gave them flexibility to micro plan around these challenges. As one of the team members put it in one of the meetings - "I should tell you, as we look at whether Lean helped us on the project or not, that we have made this progress despite various challenges only because of Lean. I do not know what it would have been like if Lean was not implemented"

\subsubsection{Challenges in implementing Lean in Design and Procurement phases}

Lean construction principles have been applied to the construction processes in various projects in this country. However, introduction of Lean philosophy in the design and procurement phases of the project posed significant challenges. The Design work in this project was outsourced and complex workflow processes involving several end-user groups, the internal coordination team and the multi-location design agency resulted in several iterations. The weekly look ahead plans and constraints identification were not easy for the design and procurement teams. The Big Room meetings mandated these team members to give commitments on certain aspects of the design. The weekly lookahead plans did help them to micro plan certain activities but as the week progressed, the team members realized that the promises given might not be fulfilled due to unanticipated iterations and design changes. Similar problems were encountered with the procurement phase, where standard operating procedures for procurement as per Lean concepts were not set before the project commenced. Unanticipated activities such as extended negotiations with vendors, etc. made it difficult for the procurement teams to keep up their promises. Such procedural aspects challenged the appropriateness of Lean implementation in the Design and Procurement phases of Lean implementation. At present methods for improving the processes of introduction and mainstreaming Lean in design and procurement are still being debated by the teams.

\subsubsection{Challenges in the Involvement of different Divisions with their own individual Processes}

The other divisions of the organisation apart from the construction division had their own project management systems, such as the Theory of Constraints and initially found it difficult to accept and adopt the Lean philosophy. This resulted in many debates and disengagement to some extent. However, on observing the Lean processes involved over time, they understood the many similarities in the approaches and came around to accepting the Lean concepts as being more comprehensive and started practicing Lean quite effectively.

\subsection{Organizational behaviour and culture}

The behavioural and cultural aspects turned out to be the most challenging in terms of sustaining the transformation to Lean over a longer period. The initial momentum of the novel philosophy started slowing down after some time. The age-old habits of the team members began to resurface. The prevalent organisational culture was a friendly one where team members were more concerned about avoiding conflicts. While this culture maintained a pleasant working environment in the organisation and the project, it impeded the team members from discussing the feasibility, etc frankly and effectively. 
As the project progressed, Lean implementation forced the members to come out of this comfort zone and discuss the issues more frankly and point out the constraints posed by other team members. Sustaining this open culture became a challenge in the long term on the project and prompted organisation-wide initiatives to change this culture.

\section{STRATEGIES THAT FACILITATE MAINSTREAMING OF LEAN CULTURE IN THE ORGANISATION}

As the mentoring coach observed the organisation while it was trying to sustain the Lean culture developed, some key findings which can be applied to other projects were noted. These findings are discussed below.

\subsection{The role of Lean steering team}

The role of a Lean steering or champion team is very important in mainstreaming Lean philosophy. This team should be in a position to gauge the morale of the working team players and persist with the changes till the working teams realized the benefits of Lean implementation. The role of this team is also crucial in realizing how various tools related to Lean construction can be implemented in the organisation in an effective manner. The team could decide on which tools to implement and also on the timing of such implementations to maximise the gains achieved by the implementation of Lean on projects. An important point in this aspect is the continuity of personnel who are part of this team. The organisation could, as far as possible, ensure that certain key persons continue on this team to improve the consistency of Lean implementation over time.

\subsection{The role of demonstrable initial successes}

It helps to have some demonstrable initial successes to sustain Lean over time. The use of the simpler tools such as 5S, Work Sampling, First Run Studies, etc help people understand Lean processes on a hands-on basis and can be useful to demonstrate early gains. The initial successes help the team members build confidence on subscribing to the change and help mainstreaming the new philosophy.

\subsection{The role of HR training}

A crucial role is played by Human Resources training in mainstreaming Lean construction. The HR teams can help facilitate the behavioural change which is required by the project teams as the organisation transitions to the new culture. The HR trainings could also aim at addressing gaps in trust and encourage an open and honest culture. In this process, they could re-emphasize the vision and goals of the organisation and how transforming to the new paradigm helps in achieving those goals. The trainings should be structured in such a way that these principles are instilled in all the employees early in their career in the organisation. Reinforcing trainings which build on the older trainings play a crucial role in the internalization of Lean culture among employees and mainstreaming the Lean culture in the organisation. The mentoring coach observed that such HR trainings might also be required for the vendors and contractors who are part of the projects.

\subsection{The role of technology}

The organisation could substantially invest in technologies which would help in mainstreaming Lean. This might involve software tools that facilitate coordination and 
construction such as comprehensive modelling tools like Digital workflow models, Visualization and BIM enabled-Lean. The mentoring coach had observed significant improvement in the effectiveness of the PPC measures when the organisation introduced special software to automate the processes of reporting. Such simple technology tools might help Lean implementation in a big way.

\subsection{The role of top management buy-in}

The role played by top management is extremely critical in the mainstreaming. The top management could make it amply clear that Lean would be the main or only way of delivering projects in the organisation and should take visible actions to this effect. Such actions by the top management will facilitate the shifting to the new philosophy and aid in changing the organisational culture. Further, sustained involvement and motivation by the top management is invaluable in sustained Lean implementation.

\subsection{The role of a Lean Mentor/ Coach}

The availability of an experienced Lean mentor or coach can help in maintaining sustained practice of Lean concepts and help in charting mid-course corrections to ensure sustained Lean implementation. The various problems encountered in the project as mentioned earlier sometimes had a negative impact on the overall PPC trend in the Last Planner process used. (Fig. 1). However, with the guidance of the Lean coach and ever-growing permeation of Lean culture in the various teams, the negative trends were rectified.

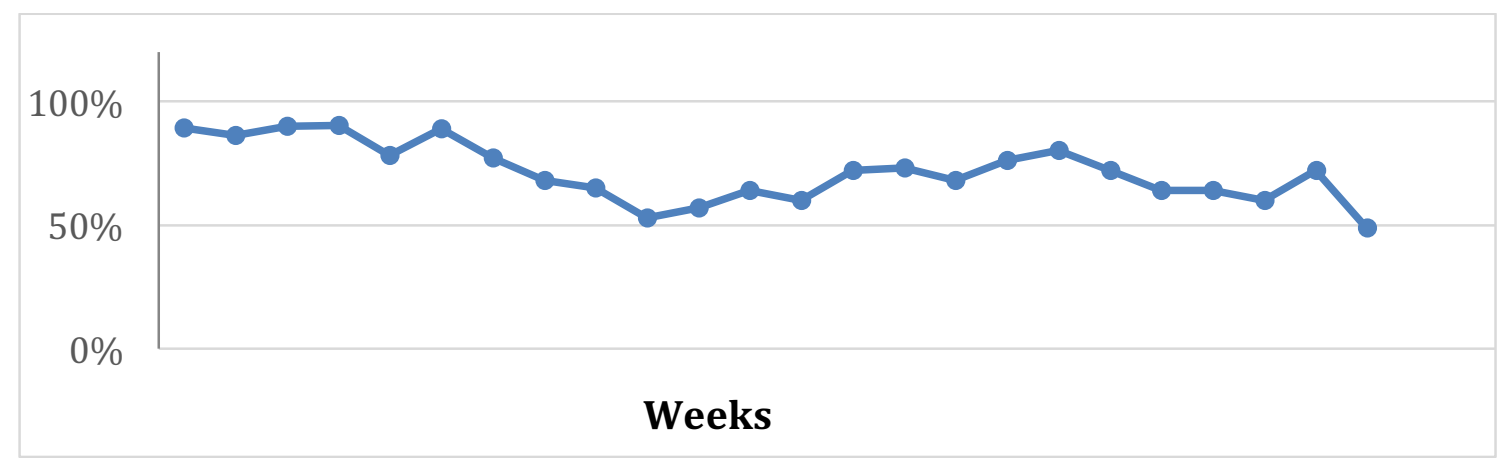

Fig.1 Variation of overall PPC over time

\section{IMPLICATIONS AND CONCLUSIONS}

The study illustrates some key aspects related to organisational structure, processes and culture which can pose significant challenges or enable mainstreaming while implementing Lean construction in a project or in an organisation. It was noted that changing the organisational structure or introducing tools did not pose significant challenges if the top management was fully subscribed. However, a change in processes is relatively difficult to achieve in an organisation. Finally, the organisational culture is a significant factor in the transformation to the new paradigm. Though the team members adopt the new philosophy in the short term, the deep seated beliefs would resurface over time and if not re-aligned to the new philosophy continuously, would pose significant challenges in transforming to Lean culture. Strategic planning by the top management is required to surmount these challenges. Such strategies might include demonstrating the 
initial successes achieved with Lean philosophy to reinforced trainings to transform the organisational culture. The use of technology and tools such as Digital workflows, automated reporting and alerting tools, etc. might help in improving the effectiveness of Lean and also help transform the team. Top managements should strategically plan such interventions to effectively mainstream Lean culture in an organisation.

\section{REFERENCES}

Raghavan, N. (2015). "Implementing Lean Concepts in Indian Construction Sites- A Trial and its Outcome." Indian Lean Construction Conference, Mumbai, 39-52.

Raghavan, N., Kalidindi, S., Mahalingam, A., Varghese, K., and Ayesha, A. (2014). "Implementing Lean Concepts on Indian Construction Sites: Organisational Aspects and Lessons Learned." Proceedings of the 22nd annual conference of the International Group for Lean Construction, Oslo, 1181-1190.

Ballard, G. (2015). "Bringing Lean into Indian Construction Industry." Proceedings of the Indian Lean Construction Conference, Mumbai, 1-13.

Raghavan, N., Venkata S.K.D., Mahalingam, A., Varghese, K. (2016). "Introducing Lean Construction Philosophy in E-P-C Phases of a Large Industrial Project", Proceedings of the 24th annual conference of the International Group for Lean Construction, Boston

Keiser, J. A. (2012) "Leadership and Cultural Change: Necessary Components of a Lean Transformation". Proceedings of the 20th annual conference of the International Group for Lean Construction, San Diego

Landsberger, H. A. (1958). "Hawthorne Revisited: Management and the Worker, Its Critics, and Developments in Human Relations in Industry". Cornell University Press 